\title{
Síndrome Coronária Aguda sem Supradesnível de ST de Alto Risco: a Mortalidade Intra-Hospitalar é Proporcional ao Retardo Para a Intervenção
}

\author{
Carlos Augusto Homem de Magalhães Campos' ${ }^{1}$, Pedro A. Lemos ${ }^{1}$, Expedito E. Ribeiro', \\ João L. A. A. Falcão', Marco A. Perin'1, Luiz J. Kajita', Antonio Esteves Filho'1, \\ Marcus N. da Gama', Pedro E. Horta', Gilberto G. Marchiori', Andre G. Spadaro', \\ Paulo R. Soares ${ }^{1}$, Silvio Zalc ${ }^{1}$, Eulógio E. Martinez ${ }^{1}$
}

\section{RESUMO}

Fundamentos: Estudos recentes mostram que uma abordagem invasiva rotineira para pacientes com síndrome coronária aguda sem supradesnivelamento do segmento ST diminui eventos em relação a uma abordagem conservadora, mas o tempo ideal para esta abordagem ainda é motivo de debate. Método: No período de maio/2003 a novembro/ 2005, 466 pacientes com infarto agudo do miocárdio, sem supradesnível do segmento ST, foram submetidos à intervenção coronária percutânea, em nossa instituição. Excluímos aqueles pacientes com instabilidade hemodinâmica à admissão. Os pacientes foram divididos em dois grupos, de acordo com o tempo entre a admissão e a realização da angioplastia: 1) Grupo Precoce (intervenção $\leq 6$ horas) com 152 pacientes e 2) Grupo Tardio (intervenção >6 horas) com 314 pacientes. Foram analisados os dados do procedimento e a mortalidade intra-hospitalar. Resultados: Os dois grupos foram semelhantes quanto às suas características clínicas, exceto pela maior freqüência de cirurgia coronária prévia no Grupo Tardio. Pacientes no Grupo Precoce foram tratados com uma mediana de 3 horas (intervalo interquartil 2-4 horas) e, no Grupo Tardio, após 23 horas (intervalo interquartil 14-48 horas). Pacientes tratados precocemente apresentaram mortalidade intrahospitalar significativamente menor que os pacientes do Grupo Tardio $(0,7$ vs. 4,8\%; $p=0,02)$. À análise multivariada, idade, insuficiência cardíaca e tempo de tratamento foram identificados como preditores independentes de óbito hospitalar. Conclusão: A realização de angioplastia precoce

\section{SUMMARY}

High Risk Unstable Coronary Syndrome: In-Hospital Mortality is Increased With Delayed Intervention

Background: Several studies have shown that routine invasive strategies reduce major events compared to a conservative strategy for patients with acute coronary syndrome without ST elevation. However, the optimal time to institute this approach is still debatable. Methods: From May 2003 to November 2005, 466 patients with myocardial infarction without ST elevation, excluding patients with hemodynamic instability, were treated by percutaneous coronary interventions $(\mathrm{PCl})$ in our hospital. Patients were divided in 2 groups according to time from admission to $\mathrm{PCl}$ : 1) Early Group ( $\leq 6 \mathrm{~h}$ ) involving 152 patients and 2) Late Group $(>6$ h) including 314 patients. Procedure data and in-hospital mortality were analyzed. Results: Baseline clinical characteristics were similar in both groups, except for prior CABG that was more frequent in late group. Median time from admission to $\mathrm{PCl}$ was 3 hours (interquartile interval: 2-4hours) in the Early Group and 23 hours (interquartile interval: 14-48hours) in the Late Group. In-hospital mortality was significantly reduced in the Early Group (0.7 vs. 4.8\%; $\mathrm{p}=0.02)$. Age, heart failure and time from admission to $\mathrm{PCl}$ were independent predictors of in-hospital mortality by multivariate analysis. Conclusion: Early PCI for myocardial infarction without ST elevation seems to reduce the inhospital mortality in the daily practice. The clinical impact

\footnotetext{
1 Serviço de Hemodinâmica e Cardiologia Intervencionista do Instituto do Coração - InCor Hospital das Clínicas, Faculdade de Medicina da Universidade de São Paulo, São Paulo, SP. Correspondência: Dr. Pedro A. Lemos. Av. Dr. Enéas de Carvalho Aguiar, 44 - Bloco I, $3^{\circ}$ andar, Hemodinâmica - São Paulo - SP - Brasil CEP 05403-000 - Tel +55-11-30695212 - Fax +55-11-3069 5286 E-mail: pedro.lemos@incor.usp.br

Recebido em: 31/6/2007 • Aceito em: 13/8/2007
} 
Campos CAHM, et al. Síndrome Coronária Aguda sem Supradesnível de ST de Alto Risco: a Mortalidade Intra-Hospitalar é Proporcional ao Retardo Para a Intervenção. Rev Bras Cardiol Invas 2007; 15(3): 244-248.

parece reduzir o risco óbito intra-hospitalar em pacientes com infarto agudo do miocárdio sem supradesnível do segmento ST tratados no dia-a-dia. O impacto clínico da implementação de protocolos de tratamento acelerado para estes pacientes deve ser avaliado no ambiente de estudos randomizados.

DESCRITORES: Coronariopatia. Infarto do miocárdio. Angioplastia transluminal percutânea coronária. Contenedores.

$\mathrm{A}$ s síndromes coronárias agudas sem elevação do segmento ST representam um grande problema de saúde pública, sendo o principal motivo de atendimento de urgência e internação no mundo ocidental $^{1}$ e podendo atingir uma mortalidade de até $12 \%$ nos primeiros 6 meses $^{2}$.

De modo geral, evidências sugerem que uma estratégia terapêutica invasiva precoce é a melhor abordagem para essa condição ${ }^{3-6}$. No entanto, o momento ideal para a revascularização (percutânea ou cirúrgica) se mantém controverso.

O presente estudo, portanto, objetiva avaliar o impacto do tempo de retardo até o tratamento sobre a mortalidade intra-hospitalar, em pacientes com síndrome coronária aguda de alto risco, tratados com intervenção coronária percutânea.

\section{MÉTODO}

O presente estudo constitui uma série observacional de 466 pacientes consecutivos, incluídos prospectivamente entre maio de 2003 e novembro de 2005, em um centro de atenção terciária à saúde no Brasil. Foram incluídos pacientes com infarto agudo do miocárdio sem supradesnível de ST (angina de peito em repouso ou progressiva, ou equivalentes anginosos, associado a qualquer elevação de CKMB massa e/ou troponina I) e que foram submetidos a intervenção coronária percutânea.

No eletrocardiograma de 12 derivações, não poderia haver supradesnível persistente do segmento ST superior a $1 \mathrm{mV}$ em duas derivações contíguas ou bloqueio de ramo esquerdo novo. Também foram excluídos pacientes que, à admissão, apresentavam instabilidade hemodinâmica ou isquemia refratária, e que foram submetidos ao estudo hemodinâmico em regime de emergência pela gravidade extrema de sua condição clínica. Consideramos isquemia refratária, a presença de angina de peito, apesar de máximo tratamento medicamentoso, associada à alteração eletrocardiográfica sugestiva de isquemia coronária. Instabilidade hemodinâmica foi considerada como edema pulmonar, com necessidade de ventilação mecânica assistida, arritmia ventricular complexa sustentada ou choque cardiogênico.

Os pacientes foram divididos em dois grupos, de of this accelerated invasive strategy needs to be evaluated in randomized trials.

DESCRIPTORS: Coronary disease. Myocardial infarction. Angioplasty, transluminal, percutaneous coronary. Stents.

acordo com o tempo entre a admissão e a realização da angioplastia:

- Grupo Precoce: onde a intervenção ocorreu em um período menor ou igual a 6 horas (152 pacientes);

- Grupo Tardio: onde a intervenção ocorreu em um período maior do que 6 horas (314 pacientes).

Os dados da evolução intra-hospitalar foram coletados por médicos treinados, durante a internação índice, seguindo o preenchimento de formulários previamente padronizados. A coleta incluiu características clínicas, resultados de exames laboratoriais, dados do procedimento invasivo (desde tempo até características angiográficas e da intervenção) e evolução clínica até a alta hospitalar.

As características clínicas, os padrões do tratamento (medicações utilizadas antes da intervenção e o procedimento de revascularização) e a mortalidade intrahospitalar foram comparados entre os grupos de intervenção precoce e tardia. As variáveis contínuas foram descritas como médias e desvio-padrão ou medianas e intervalo interquartil (25ㅇ e $75^{\circ}$ percentis). As variáveis categóricas foram descritas como freqüência. As variáveis contínuas foram comparadas com testes de Wilcoxon, enquanto que as variáveis categóricas foram comparadas com teste do Qui-Quadrado.

Em nossa análise, nós ajustamos os dados de nossa amostra para as várias características clínicas e angiográficas, incluindo: idade, sexo, hipertensão arterial sistêmica, diabetes mellitus, dislipidemia, insuficiência cardíaca, insuficiência renal crônica dialítica, insuficiência arterial periférica, infarto do miocárdio prévio, acidente vascular cerebral prévio, intervenção coronária percutânea prévia, cirurgia de revascularização miocárdica prévia, tabagismo, antecedente familiar, uso de stent, número de artérias coronárias com obstruções superiores a $50 \%$ da luz e qual o vaso tratado pela intervenção. Todas estas covariáveis foram incluídas em uma análise de regressão.

\section{RESULTADOS}

No Grupo Precoce (152 pacientes tratados com tempo inferior a 6 horas), o tempo entre a admissão 
Campos CAHM, et al. Síndrome Coronária Aguda sem Supradesnível de ST de Alto Risco: a Mortalidade Intra-Hospitalar é Proporcional ao Retardo Para a Intervenção. Rev Bras Cardiol Invas 2007; 15(3): 244-248.

e o tratamento teve mediana de 3 horas (intervalo interquartil 2-4 horas), enquanto no Grupo Tardio (314 pacientes com um período superior a 6 horas), a mediana do tempo de retardo foi de 23 horas (intervalo interquartil 14-48 horas), conforme ilustra a Figura 1. Não houve diferença significativa das características clínicas entre pacientes tratados precocemente e tardiamente, exceto pela maior presença de pacientes com cirurgia de revascularização miocárdica prévia no grupo da intervenção tardia (Tabela 1). Os pacientes tinham idade média próxima a 65 anos, um terço era do sexo feminino, cerca de $30 \%$ tinham diabetes mellitus e $15 \%$ insuficiência cardíaca. As características angiográficas dos dois grupos foram semelhantes, com $70 \%$ dos pacientes com doença multivascular e com abordagem terapêutica, principalmente na artéria descendente anterior $(42,1 \%)$. O tratamento medicamentoso, administrado antes da intervenção coronária, foi diferente entre os grupos por um maior uso de inibidores da enzima conversora da angiotensina (39\% versus 53\%, $p=0,007)$ e estatinas $(35,6 \%$ e $48,7 \%, p=0,009)$; conforme mostrado na Tabela 2.

A evolução intra-hospitalar foi significativamente diferente entre os grupos estudados. Pacientes tratados precocemente apresentaram mortalidade hospitalar significativamente menor que o Grupo Tardio $(0,65$ vs. $4,77 \%, \mathrm{p}=0,02)$. À análise multivariada, mesmo após ajuste estatístico para os fatores listados na Tabela 1, o Grupo Tardio ainda persiste com maior risco de óbito intra-hospitalar (OR ajustada 7,8; IC 95\%: $1,0-60,7 ; p=0,05)$. Além do tempo até a intervenção, encontramos como preditores independentes de mortalidade: idade [OR ajustada 1,1; IC95\%: 1.01-1.1; $p<0,01$ )] e a presença de insuficiência cardíaca [OR ajustada 4,5; IC95\%: 1,5-13,6; $\mathrm{p}<0,01)$ ].

\section{DISCUSSÃO}

O dado mais importante obtido neste estudo é que os pacientes de uma população brasileira do "mun-

TABELA 1

Características clínicas e angiográficas de base da população do presente estudo

\begin{tabular}{|c|c|c|c|}
\hline & Grupo Precoce $(n=152)$ & Grupo Tardio $(n=314)$ & $\mathbf{p}$ \\
\hline Idade, anos & $65,3 \pm 12$ & $64,3 \pm 12$ & 0,4 \\
\hline Sexo masculino, \% & 67,5 & 70,7 & 0,5 \\
\hline Diabetes mellitus, \% & 30,2 & 27,6 & 0,5 \\
\hline Hipertensão arterial, \% & 85,1 & 84,2 & 0,8 \\
\hline Dislipidemia, \% & 70,1 & 76,6 & 0,2 \\
\hline Tabagismo, \% & 29 & 29 & 1 \\
\hline Antecedente familiar, \% & 34,6 & 44,2 & 0,8 \\
\hline AVC, $\%$ & 3,4 & 4 & 1 \\
\hline IRC dialítica, \% & 5,3 & 2 & 0,08 \\
\hline IVP, \% & 7,33 & 4,95 & 0,4 \\
\hline IAM prévio, \% & 33,6 & 40,3 & 0,17 \\
\hline $\mathrm{ICC}, \%$ & 15 & 14,7 & 1 \\
\hline ICP prévia, \% & 22,2 & 18,6 & 0,38 \\
\hline RM prévia, \% & 13,4 & 24,8 & 0,05 \\
\hline \multicolumn{4}{|c|}{ Coronárias acometidas com obstrução superior a 50\% } \\
\hline Uniarterial, \% & 34,5 & 27,2 & 0,2 \\
\hline Biarterial, \% & 27,7 & 34,4 & 0,2 \\
\hline Triarterial, \% & 37,8 & 38,4 & 0,3 \\
\hline \multicolumn{4}{|l|}{ Vaso Tratado } \\
\hline $\mathrm{TCE}, \%$ & 0,6 & 1,59 & 0,3 \\
\hline DA, \% & 42,1 & 38,8 & 0,5 \\
\hline $\mathrm{CX}, \%$ & 30,2 & 30 & 1 \\
\hline$C D, \%$ & 30 & 28,3 & 0,6 \\
\hline Uso de stent, \% & 94,7 & 93 & 0,6 \\
\hline Lesões Abordadas/Paciente & $1,36 \pm 0,6$ & $1,37 \pm 0,7$ & 0,6 \\
\hline
\end{tabular}

Abreviações: AVC - acidente vascular cerebral; IRC - insuficiência renal crônica; IVP - insuficiência vascular periférica; IAM - infarto agudo do miocárdio; ICC - insuficiência cardíaca; ICP - intervenção coronária percutânea; RM - cirurgia de revascularização miocárdica; TCE - tronco de coronária esquerda; DA - artéria descendente anterior; CX - artéria circunflexa; CD - artéria coronária direita. 
Campos CAHM, et al. Síndrome Coronária Aguda sem Supradesnível de ST de Alto Risco: a Mortalidade Intra-Hospitalar é Proporcional ao Retardo Para a Intervenção. Rev Bras Cardiol Invas 2007; 15(3): 244-248.

TABELA 2

Medicações prescritas para os pacientes previamente à intervenção

\begin{tabular}{lcccccc}
\hline & \multicolumn{2}{c}{ Grupo Precoce } & & \multicolumn{3}{c}{ Grupo Tardio } \\
\cline { 2 - 3 } Medicações & $\mathbf{n}$ & $\mathbf{\%}$ & & $\mathbf{n}$ & \% & $\mathbf{p}$ \\
\hline AAS & 147 & 96,7 & 308 & 98 & 0,86 \\
Antagonista de canal de cálcio & 16 & 10,5 & 30 & 9.6 & 0,74 \\
Betabloqueadores & 84 & 55,2 & 199 & 63.3 & 0,11 \\
Clopidogrel & 41 & 27 & 104 & 33.1 & 0,17 \\
Heparina de baixo peso molecular & 28 & 18,4 & 80 & 25.5 & 0,11 \\
Heparina não fracionada & 124 & 81,6 & 234 & 74,5 & 0,69 \\
Inibidores da ECA & 60 & 39,5 & 167 & 53,1 & 0,007 \\
Estatinas & 54 & 35,6 & 153 & 48,7 & 0,009 \\
Ilb/Illa & 65 & 42,8 & 151 & 48 & 0,32 \\
\hline
\end{tabular}

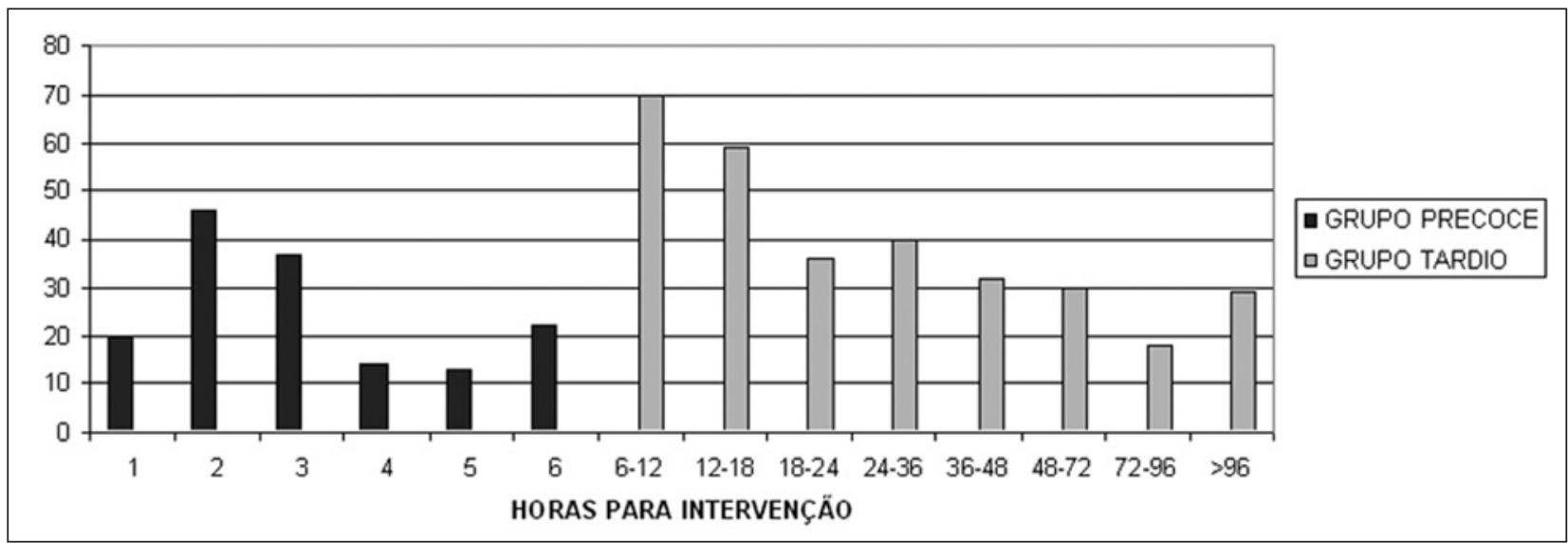

Figura 1 - Intervalo de tempo entre a admissão e a intervenção.

do real", com infarto agudo do miocárdio sem supradesnível do segmento ST, tiveram mortalidade intrahospitalar menor quando tratados mais precocemente.

A definição de "terapia invasiva precoce" possui grande divergência na literatura, podendo corresponder a um período de 6 horas (utilizado no estudo ISAR$\mathrm{COOL}^{7}$ ) ou até 48 horas (definição do estudo ICTUS ${ }^{8}$ ), obtendo conclusões distintas. Preferimos, neste estudo, definir como precoce aquele paciente tratado em um período inferior a 6 horas, já que no estudo ISARCOOL houve benefício do tratamento precoce, exceto naqueles pacientes com elevação dos marcadores de necrose miocárdica ${ }^{7}$. Como os pacientes com alteração de troponina e CKMB estão expostos a um risco maior de eventos ${ }^{9,10}$, procuramos explorar o comportamento desta população submetida à terapia de revascularização por cateter.

Apesar de não se tratar de um estudo randomizado, os grupos (precoce e tardio) foram bem semelhantes quanto a suas características clínicas. Houve apenas maior freqüência de pacientes submetidos a cirurgia de revascularização prévia no grupo tardio $(24,8 \%$ vs. $13,4 \%, p=0,05$ ) e tendência a diferença na freqüência de portadores de insuficiência renal crônica dialítica no grupo precoce $(5,3 \%$ vs. $2 \%, p=0,08)$. Contudo, estas características clínicas não foram identificadas como preditores de óbito à análise multivariada.

Houve diferença significativa entre os grupos quanto ao tipo de tratamento medicamentoso administrado antes do procedimento de revascularização. O grupo tardio recebeu mais freqüentemente estatinas $(48,7 \%$ vs. $35,6 \%, \mathrm{p}<0,01)$ e inibidores da ECA $(53,1 \%$ vs. $39,5 \%, p<0,01)$. Existem evidências de que o tratamento com estatinas produza um efeito antiinflamatório vascular, melhore a função endotelial e diminua a formação de trombos ${ }^{11}$. A utilização dos inibidores da ECA já mostrou diminuir mortalidade na terapia conjunta das síndromes coronárias agudas ${ }^{12}$. Porém, a maior utilização destas drogas não conseguiu mostrar seus benefícios nesta população.

A sobrevida dos pacientes infartados tratados com intervenção coronária percutânea foi significativamen- 
te influenciada por dois fatores clínicos de conhecida importância prognóstica para outros subgrupos ${ }^{13,14}$ : idade [OR 1.067, 95\% IC (1.01-1.1); $p<0,01]$ e a presença de insuficiência cardíaca [OR 4,5, 95\% IC (1,5-13,6); $p<0,01]$. Estes achados reforçam um cuidado diferencial que tais subgrupos merecem, onde devemos considerar um tratamento agressivo com o intuito de reduzir seu perfil de risco.

Na presente série, foram estudados pacientes relativamente complexos, incluindo portadores de comorbidades graves, como insuficiência renal dialítica (3\%), insuficiência cardíaca $(14,6 \%)$, infarto agudo do miocárdio prévio $(38 \%)$ e insuficiência arterial periférica $(5,6 \%)$. O grupo reflete o dia-a-dia da prática clínica de centros cardiológicos terciários. É possível que o espectro de alta gravidade dos pacientes incluídos pode ter exarcebado as diferenças observadas entre os grupos Precoce e Tardio. Portanto, é importante ressaltar que, em centros com atendimento voltado a pacientes com menor perfil de risco, a diferença entre as duas abordagens (precoce vs. tardia) pode não ser tão marcada. E o impacto destas estratégias no prognóstico de pacientes de menor risco deve ser avaliado em estudo específico neste contexto clínico.

\section{CONCLUSÃO}

A realização de angioplastia precoce parece reduzir o risco de óbito intra-hospitalar em pacientes com infarto agudo do miocárdio sem supradesnível do segmento ST tratados no dia-a-dia. O impacto clínico da implementação de protocolos de tratamento acelerado para estes pacientes deve ser avaliado no ambiente de estudos randomizados.

\section{REFERÊNCIAS BIBLIOGRÁFICAS}

1. Bertrand ME, Simoons ML, Fox KA, Wallentin LC, Hamm CW, McFadden E, et al. Management of acute coronary syndromes in patients presenting without persistent STsegment elevation. Task Force on the Management of Acute Coronary Syndromes of the European Society of Cardiology. Eur Heart J. 2002;23(23):1809-40.

2. Fox K, Goodman S, Klein W, Brieger D, Steg P, Dabbous $\mathrm{O}$, et al. Management of acute coronary syndromes. Variations in practice and outcome: findings from the Global Registry of Acute Coronary Events (GRACE). Eur Heart J. 2002;23(15):1177-89.

3. FRISC II Investigators. Invasive compared with non-invasive treatment in unstable coronary artery disease: FRISC II prospective randomised multicentre study. Lancet. 1999;354(9180): 708-15.

4. Wallentin L, Lagerqvist B, Husted S, Kontny F, Stahle E, Swahn E. Outcome at 1 year after an invasive compared with a non-invasive strategy in unstable coronary-artery disease: the FRISC II invasive randomised trial. FRISC II Investigators. Fast Revascularisation during Instability in Coronary artery disease. Lancet. 2000;356(9223):9-16.

5. Clayton TC, Pocock SJ, Henderson RA, Poole-Wilson PA, Shaw TR, Knight R, et al. Do men benefit more than women from an interventional strategy in patients with unstable angina or non-ST-elevation myocardial infarction? The impact of gender in the RITA 3 trial. Eur Heart J. 2004;25(18):1641-50.

6. Cannon CP, Weintraub WS, Demopoulos LA, Vicari R, Frey MJ, Lakkis $\mathrm{N}$, et al. Comparison of early invasive and conservative strategies in patients with unstable coronary syndromes treated with the glycoprotein IIb/IIla inhibitor tirofiban. N Engl J Med. 2001;344(25):1879-87.

7. Neumann FJ, Kastrati A, Pogatsa-Murray G, Mehilli J, Bollwein $\mathrm{H}$, Bestehorn HP, et al. Evaluation of prolonged antithrombotic pretreatment ("cooling off" strategy) before intervention in patients with unstable coronary syndromes: a randomized controlled trial. JAMA. 2003;290(12):1593-9.

8. Hirsch A, Windhausen F, Tijssen JG, Verheugt FW, Cornel JH, Winter RJ, for the Invasive versus Conservative Treatment in Unstable coronary Syndromes (ICTUS) investigators. Long-term outcome after an early invasive versus selective invasive treatment strategy in patients with non-ST-elevation acute coronary syndrome and elevated cardiac troponin T (the ICTUS trial): a follow-up study. Lancet. 2007;369(9564): 827-35.

9. Hamm CW, Ravkilde J, Gerhardt W, Jorgensen P, Peheim E, Ljungdahl L, et al. The prognostic value of serum troponin $\mathrm{T}$ in unstable angina. N Engl J Med. 1992;327(3):146-50.

10. Pettersson T, Ohlsson O, Tryding N. Increased CKMB (mass concentration) in patients without traditional evidence of acute myocardial infarction. A risk indicator of coronary death. Eur Heart J. 1992;13(10):1387-92.

11. Sposito AC, Chapman MJ. Statin therapy in acute coronary syndromes: mechanistic insight into clinical benefit. Arterioscler Thromb Vasc Biol. 2002;22(10):1524-34.

12. Mukherjee D, Fang J, Chetcuti S, Moscucci M, Kline-Rogers E, Eagle KA. Impact of combination evidence-based medical therapy on mortality in patients with acute coronary syndromes. Circulation. 2004;109(6):745-9.

13. Feldman DN, Gade CL, Slotwiner AJ, Parikh M, Bergman G, Wong SC, et al. Comparison of outcomes of percutaneous coronary interventions in patients of three age groups $(<60$, 60 to 80 , and $>80$ years) (from the New York State Angioplasty Registry). Am J Cardiol. 2006;98(10):1334-9.

14. Harrell L, Schunkert H, Palacios IF. Risk predictors in patients scheduled for percutaneous coronary revascularization. Catheter Cardiovasc Interv. 1999;48(3):253-60. 